\title{
Analysis of Disc Wear Based on Trajectory Simulation in Lapping Process
}

\author{
Wen-Wen DENG ${ }^{1, a,{ }^{*}}$ and Zai-Xing ZHAO ${ }^{1, b}$ \\ ${ }^{1}$ Institute of Manufacturing Engineering, Huaqiao University, Xiamen, China

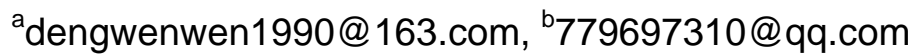

Keywords: Lapping, Processing parameters, Disc wear, Trajectory simulation

\begin{abstract}
The surface trajectory of lapping disc in lapping process was simulated in this paper. The simulation was based on the analysis of lapping disc wear trajectory by using the computer simulation technology. The law of disc wear trajectory and the influence of processing parameters were studied. The computer simulation of the disc wear trajectory actually reflects the condition of lapping disc wear in lapping process. When the RSR is an integer, the RSR determines the number of petals, and the number of the outermost petal is one. When the RSR is a fraction, the denominator of RSR fraction determines the number of the outermost petals, the numerator of RSR fraction determines the number of petal layers. In different eccentric distance, the lapping trajectory was distributed in the annular region whose width is $2{ }^{*} r_{A}\left(r_{A}\right.$ is wafer point radius). For a certain rotation speed rate, $\mathrm{n}$, the lapping trajectory of the wafer point with initial phase angle $\theta$, the trajectory turns to the angle of $n^{*} \theta$. Analyzing trajectory simulation of the disc wear has a significant guidance for designing and makign lapping disc and the actual lapping process.
\end{abstract}

\section{Introduction}

Lapping and polishing technology as an important means of precision and ultra-precision machining, is widely used in the optical element and semiconductor materials manufacturing, such as silicon nitride substrate, sapphire substrate, optical lens machining and other hard brittle materials [1]. In the lapping process, the early research indicates that tools will produce uneven wear. The partial factors of uneven wear are abrasive distribution and load distribution, resulting in a decline in the quality of the wafer surface [2]. Therefore, in order to improve the quality of the wafer surface to achieve the goal of uniform wear, the study on the wafer motion trajectory distribution relative to the lapping tool has important significance theoretically and practical referential value.

In previous studies, scholars have carried out a large number of studies on removing uniformity of the wafer material, establishing the wafer surface motion trajectory model and the model of material removal rate $[3,4]$. But the uniformity of the lapping disc wear is rarely reported. Some researchers studied the law of tool wear in plane lapping process, and revealed that the different degree of tool wear appearing in lapping processing. Meanwhile, they discovered that the law of tool wear varied with the disc surface pressure, relative rotary speed and the processing time [5]. However, the influence of the wafer parameters and other processing parameters on lapping tool wear are not considered previously. Also some researches showed that the uniformity of the lapping tool surface trajectory was able to reflect the wear of lapping tool [6,7]. Therefore, combining with the knowledge of kinematics and establishing the model of lapping disc wear trajectory, this paper is carried out a detailed analysis on the relation between the distribution of disc wear trajectory and the lapping processing parameters. Referencing the method of uniformity of material removal, the uniformity of disc wear was analyzed. Thus, based on the relationship of erosion rate and disc position, uniform wear of lapping disc can be designed and made, and the reasonable processing parameters can be selected.

\section{Lapping Disc Wear Trajectory Model}

In the lapping wafer processing, the lapping disc and wafer rotate separately with a certain velocity around the axis of rotation [8]. Fig. 1 presents that lapping disc rotates around the axis $\mathrm{O}_{1}$, and the 
wafer rotates around the axis $\mathrm{O}_{2}$, respectively. Rectangular Cartesian coordinate system was established in the motion plane, coordinate axis $\mathrm{X}_{1}$ and coordinate axis $\mathrm{X}_{2}$ were on the same straight line, origins of coordinates were $\mathrm{O}_{1}$ and $\mathrm{O}_{2}$, respectively. The distance between $\mathrm{O}_{1}$ and $\mathrm{O}_{2}$ was defined as eccentric distance, e, and one point on the wafer was defined as A. In order to analyze motion process expediently, the initial position of the $\mathrm{A}$ point is on the $\mathrm{X}$ axis hypothetically. That is, the initial phase angle is zero, the distance between $\mathrm{O}_{1}$ and $\mathrm{O}_{2}$ is $\mathrm{r}_{\mathrm{A}}$. The motion of the point $\mathrm{A}$ relative to the lapping disc is investigated. The disc rotates with an angular velocity $\omega_{\mathrm{d}}$ around the disc center $\mathrm{O}_{1}$. The wafer rotates with an angular velocity $\omega_{\mathrm{w}}$ around the disc center $\mathrm{O}_{1}$. So this relative motion is synthesis of two rotational motions.

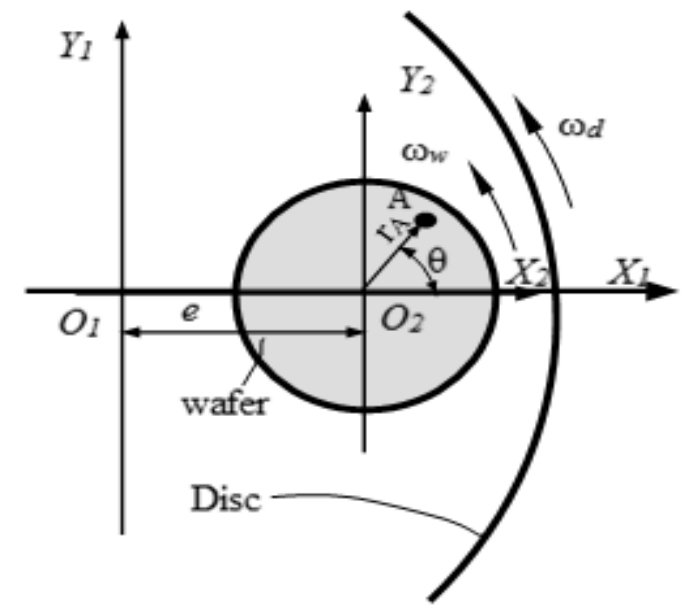

Fig. 1 The relative motion of the wafer and the lapping disc

After lapping for a period of time t, the disc rotates angle $\theta_{1}$ around its center $\mathrm{O}_{1}$, and wafer rotates angle $\theta_{2}$ around its center $\mathrm{O}_{2}$. The position of point $\mathrm{A}$ is shown as:

$$
\left\{\begin{array}{l}
\mathrm{x}=\mathrm{e} \cos \theta_{1}+r_{A} \cos \left(\theta+\theta_{2}-\theta_{1}\right) \\
y=-\mathrm{e} \sin \theta_{1}+r_{A} \sin \left(\theta+\theta_{2}-\theta_{1}\right)
\end{array}\right.
$$

That is,

$$
\left\{\begin{array}{l}
\mathrm{x}(t)=\operatorname{ecos}\left(\omega_{\mathrm{d}} \mathrm{t}\right)+r_{A} \cos \left(\theta+\omega_{\mathrm{w}} \mathrm{t}-\omega_{\mathrm{d}} \mathrm{t}\right) \\
y(t)=-\mathrm{esin}\left(\omega_{\mathrm{d}} \mathrm{t}\right)+r_{A} \sin \left(\theta+\omega_{\mathrm{w}} \mathrm{t}-\omega_{\mathrm{d}} \mathrm{t}\right)
\end{array}\right.
$$

In Eq. 1, $\mathrm{x}$ and $\mathrm{y}$ are the position of $\mathrm{A}$ in the coordinate system whose origin is in the disc center, $r_{A}$ is the distance from $A$ to the wafer center $\mathrm{O}_{2}, \theta$ is the initial location angle of $A$ on the wafer, and $\mathrm{e}$ is the distance between $\mathrm{O}_{1}$ and $\mathrm{O}_{2}$.

In Eq. 2, the rotational direction is opposite of the direction in the Fig. 1, the negative value is used as the angular velocity value. The previous research shows that the relative motion trajectory is influenced by the ratio of the lapping disc rotation speed and the wafer rotation speed, and is not related to the size of the independent rotation speed. So the rotation speed ratio (RSR) is investigated in this research.

The relative motion trajectory of the point A is a regular cycloid curve, and its curvature radius can be described as:

$$
\mathrm{p}(\mathrm{t})=\sqrt{x^{2}+y^{2}}=\sqrt{e^{2}+r_{A}^{2}+2 e r_{A} \cos \left(\theta+\omega_{\mathrm{w}} \mathrm{t}\right)}
$$

The cycloid curvature radius $\mathrm{p}(\mathrm{t})$ is a periodic function of time $t$, and its cycle is $\mathrm{T}=2 \pi / \omega_{\mathrm{w}}$. 


\section{Single-point wear Trajectory Analysis}

Disc wear trajectory is related to the RSR, which can be represented as, $\mathrm{n}=\omega \mathrm{d} / \omega \mathrm{w}$. Here, if the $\omega_{\mathrm{d}}$ and $\omega_{\mathrm{w}}$ has the same direction of rotation, $\mathrm{n}$ is positive, otherwise, $\mathrm{n}$ is negative. In simulation, the diameter of the lapping disc is $300 \mathrm{~mm}$, the diameter of the wafer is $150 \mathrm{~mm}$. Then, the influences of RSR, $\mathrm{n}$, the eccentric distance of wafer, e, the wafer point radial position, $r_{\mathrm{A}}$, and the wafer point circumferential position, $\theta$, on disc wear trajectory were studied.

The effects of the RSR, $n$. For analyzing the influence of the RSR, $n$, on the disc wear path, the wafer point radial position, $r_{A}$ is $50 \mathrm{~mm}$, the circumferential position, $\theta$ is zero, and the eccentric distance of wafer, e is $100 \mathrm{~mm}$. Fig. 2 shows the simulation results.
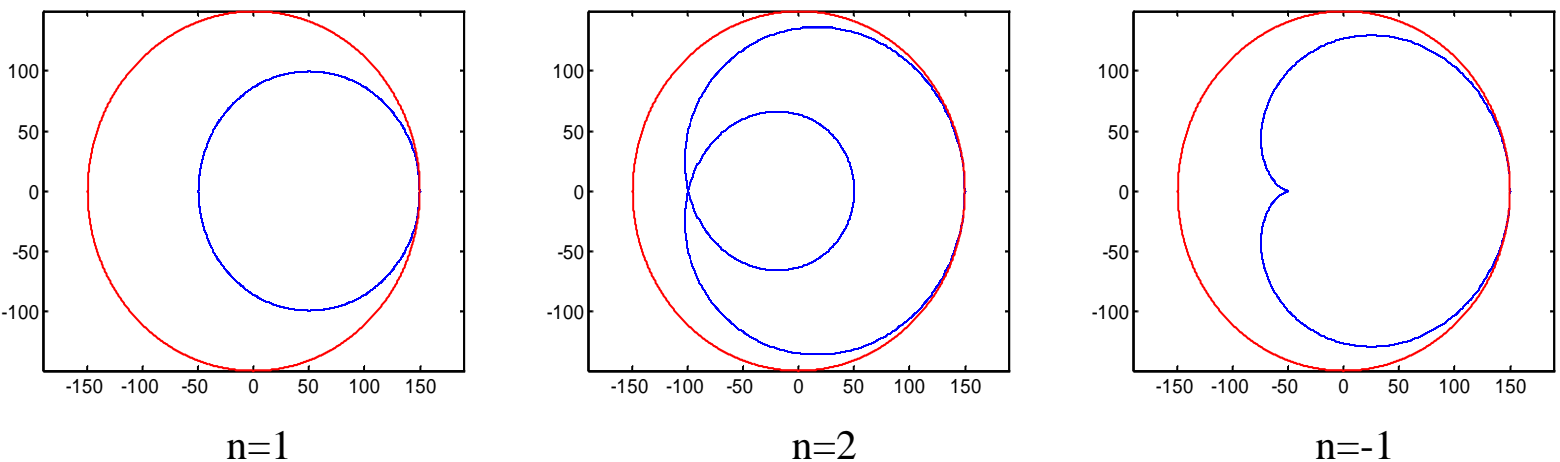

$\mathrm{n}=2$

$$
\mathrm{n}=-1
$$
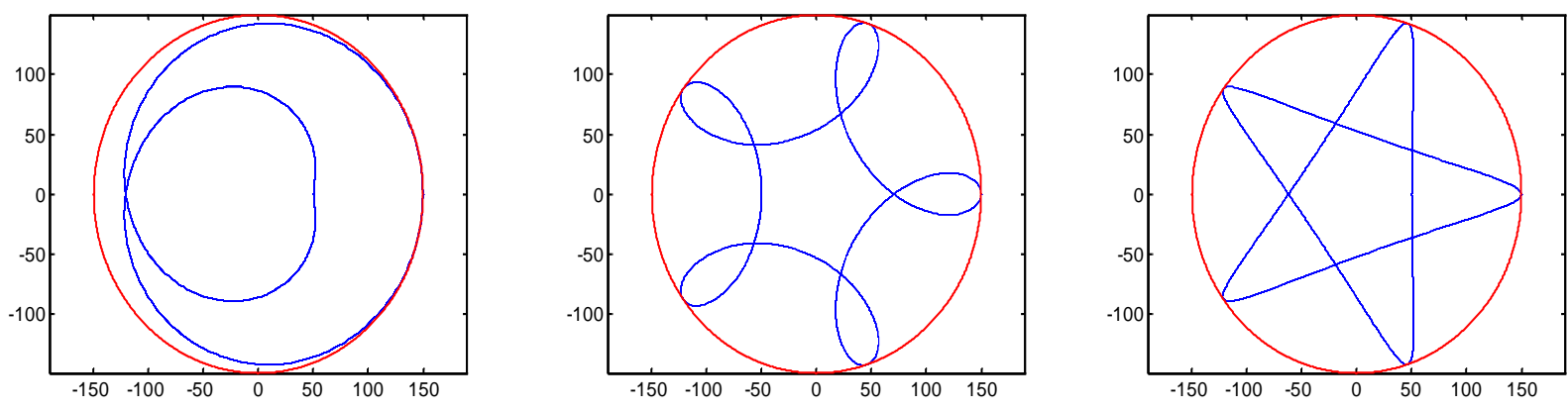

$\mathrm{n}=-2$

$\mathrm{n}=1 / 5$

$\mathrm{n}=2 / 5$
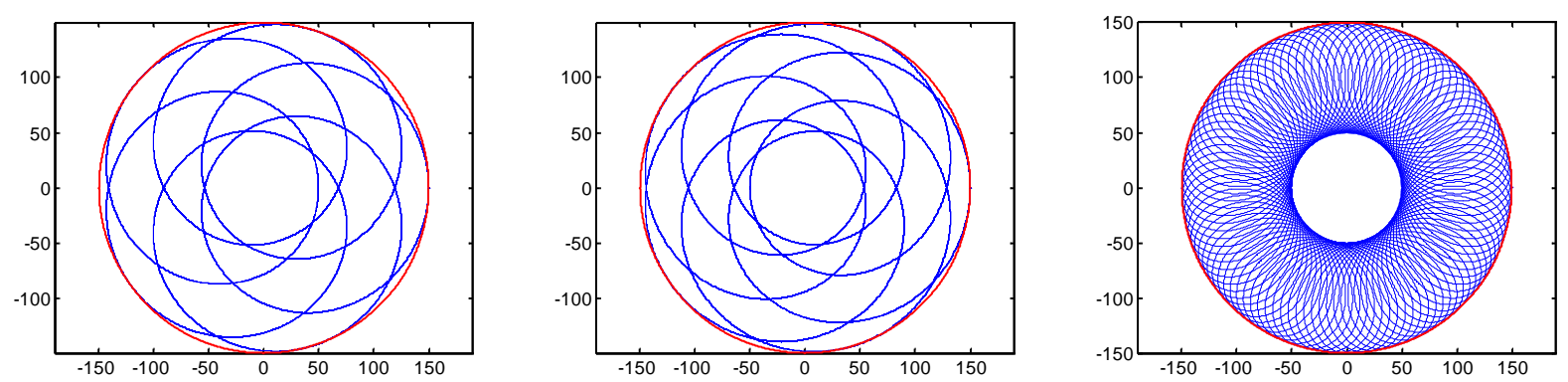

$\mathrm{n}=7 / 5$

$n=11 / 100$
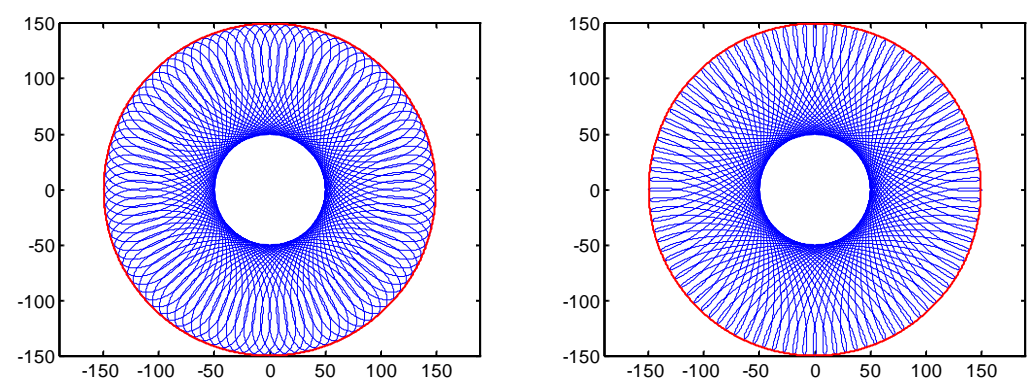

$\mathrm{n}=31 / 100$

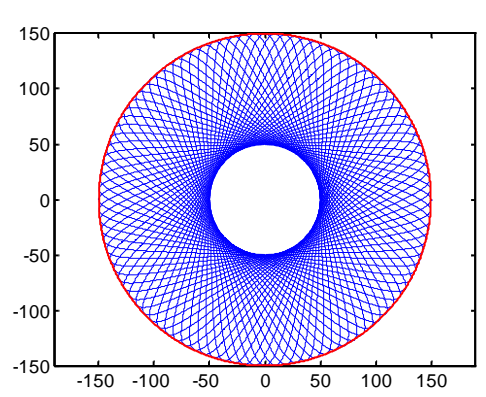

$n=41 / 100$

Fig. 2 Wafer point path on the disc with integral and fractional RSR

Fig. 2 shows the RSR has remarkable effects on the shape and distribution uniformity of the disc 
wear trajectory. When the RSR is an integer, the wafer point path shows rose lines with $\mathrm{n}$ petals, the RSR $n$ determines the number of petals, the number of petals is $n$, the number of the outermost petal is one. As to the positive RSR, the path is cycloid being similar to circle, the change of trajectory curvature is more uniform. For the negative RSR, the path is also cycloid, the curve curvature is larger, the speed changes more, and some distortion points appears in the internal.

When the RSR is a fraction, the wafer point path also shows rose lines with n petals. In addition, the denominator of RSR fraction determines the number of the outermost petals, the numerator of RSR fraction determines the number of petal layers. Compared with integral RSR, the trajectory is denser and more complex, the overlap degree of the wear trajectory is lower, the disc wear trajectory distribution uniformity is better. Through the study, it is found that when the fractional RSR is more complex, the trajectory distribution uniformity is denser and more complex, the overlap degree of the trajectory is lower, and the time reaching stable trajectory cycle is longer.

The effects of the eccentric distance, e. For analyzing the influence of the eccentric distance of wafer, e, on the disc wear path, the RSR, $n$ is $11 / 10$, the wafer point radius, $r_{A}$ is $75 \mathrm{~mm}$, and the circumferential position, $\theta$ is zero. Fig. 3 shows the simulation results.
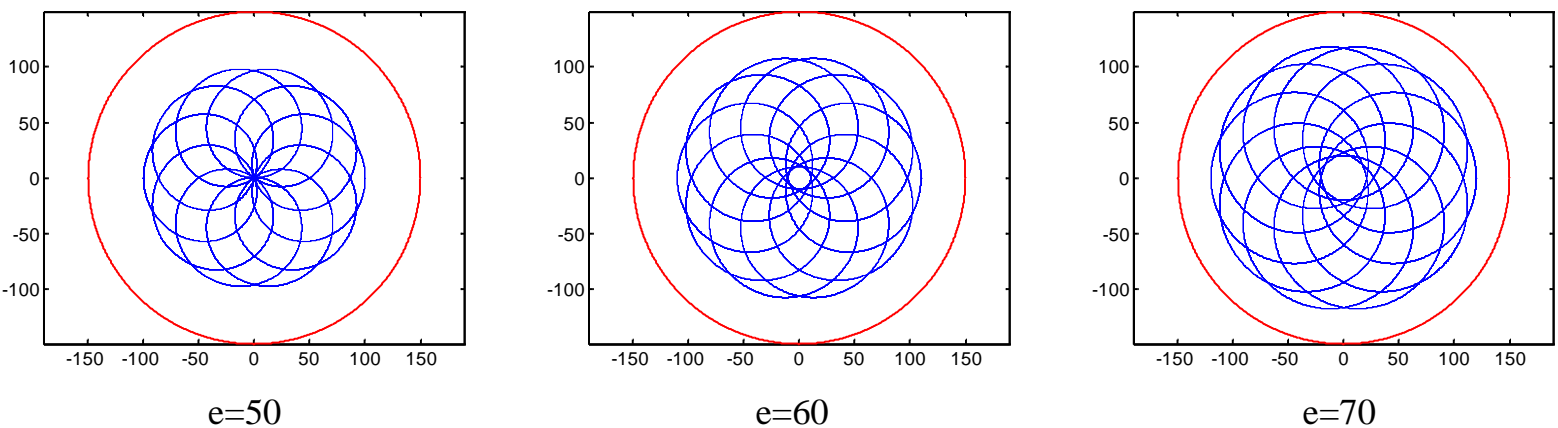

$$
\mathrm{e}=70
$$
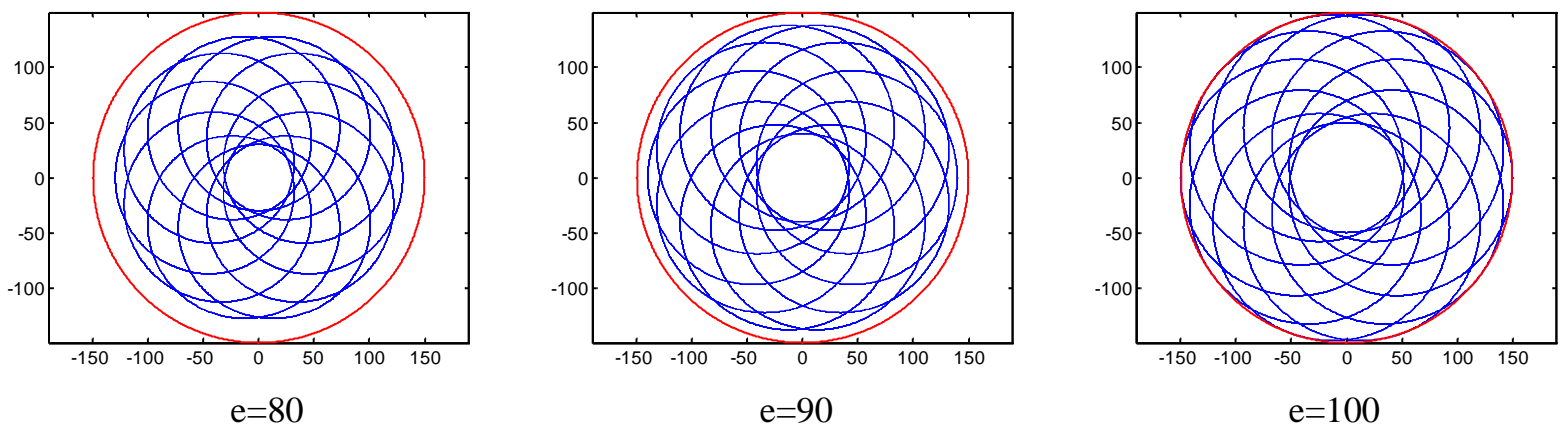

Fig. 3 Wafer point path on the disc with different eccentric distance

Fig. 3 shows, in different eccentric distance e, the lapping trajectory was distributed in the annular region whose width is $2 * \mathrm{r}_{\mathrm{A}}$. Within the region, the wear trajectory was distributed according to some laws. In other regions of the lapping disc, no contact happened to the wafer point.

The effects of the wafer point radius, $\mathbf{r}_{\mathrm{A}}$. For analyzing the influence of the wafer point radius, $r_{A}$, on the disc wear path, the RSR, $n$ is $11 / 10$, the circumferential position, $\theta$ is zero, and the eccentric distance of wafer, e is $75 \mathrm{~mm}$. Fig. 4 shows the simulation results.

Fig. 4 shows that the circular region of lapping disc contacts with wafer point with different radiuses. Wafer point radius, $\mathrm{r}_{\mathrm{A}}$, is larger, the width of lapping path region is larger and the area of lapping path region is larger. Different radiuses do not affect the cycloid shape, number, and the overlap degree.

The effects of the circumferential position, $\boldsymbol{\theta}$. For analyzing the influence of the wafer point circumferential position, $\theta$, on the disc wear path, the RSR, $\mathrm{n}$ is different numbers, the wafer point radius, $\mathrm{r}_{\mathrm{A}}$ is $75 \mathrm{~mm}$, and the eccentric distance of wafer, e is $75 \mathrm{~mm}$. Fig. 5 shows simulation results. 


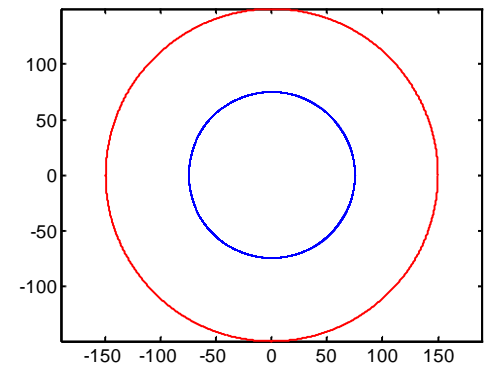

$\mathrm{r}_{\mathrm{A}}=0$

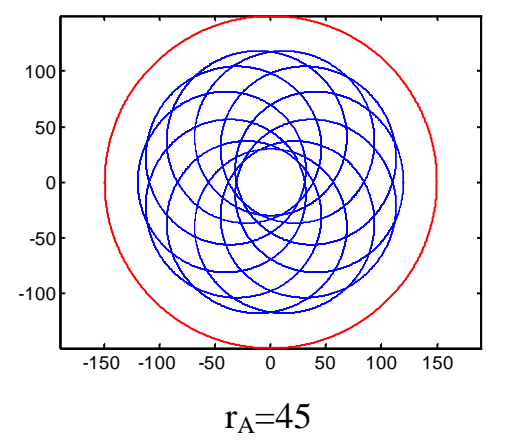

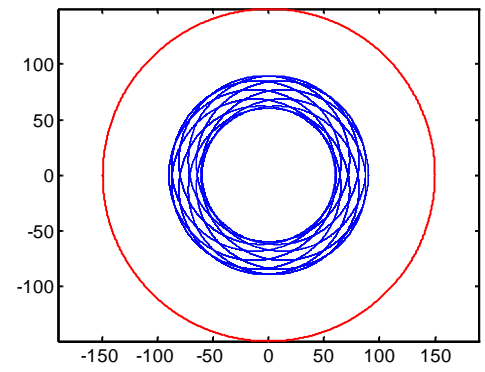

$\mathrm{r}_{\mathrm{A}}=15$

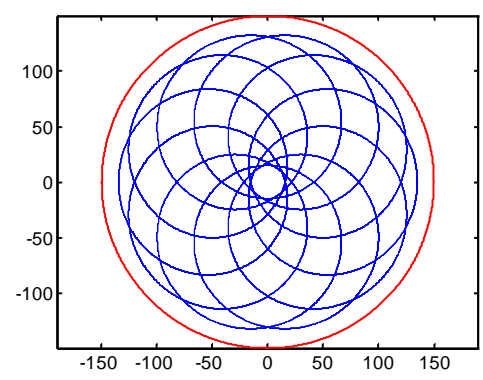

$r_{A}=60$

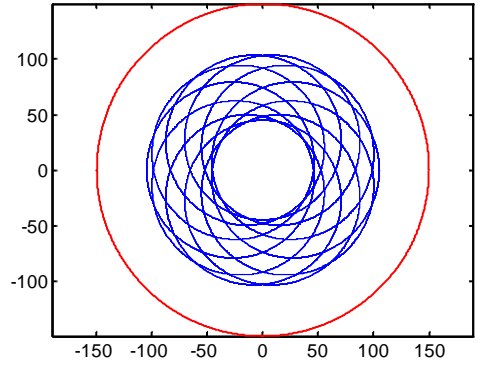

$\mathrm{r}_{\mathrm{A}}=30$

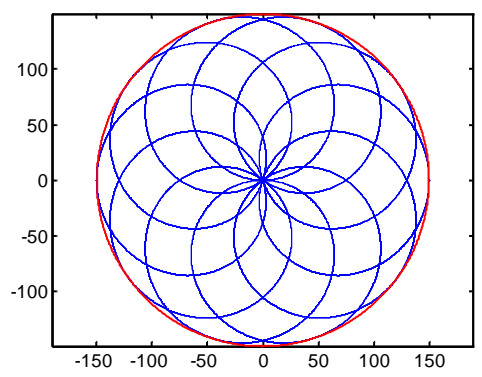

$\mathrm{r}_{\mathrm{A}}=75$

Fig. 4 Wafer point path on the disc with different wafer point radius

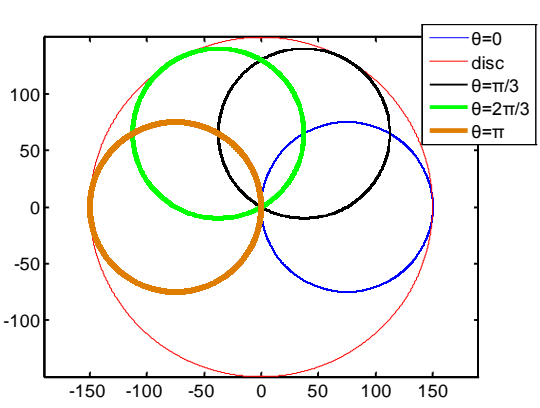

$\mathrm{n}=1$

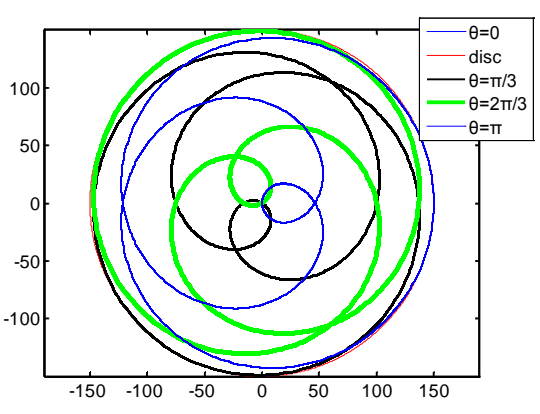

$\mathrm{n}=-2$

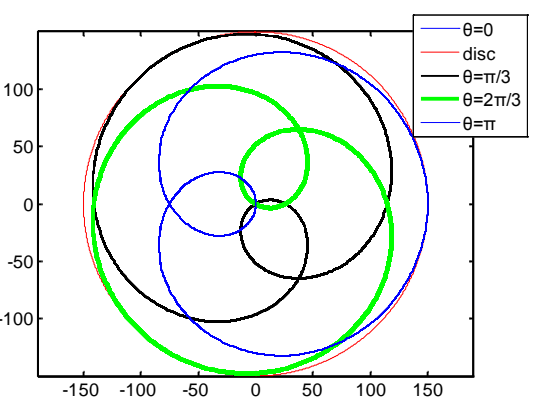

$\mathrm{n}=2$

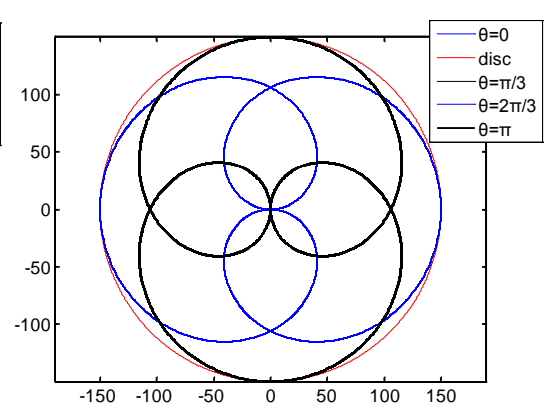

$\mathrm{n}=3 / 2$

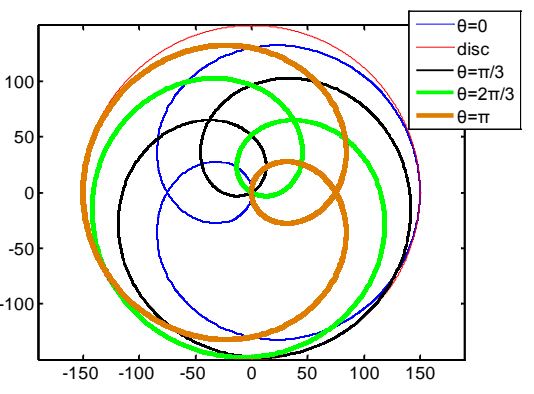

$\mathrm{n}=-1$

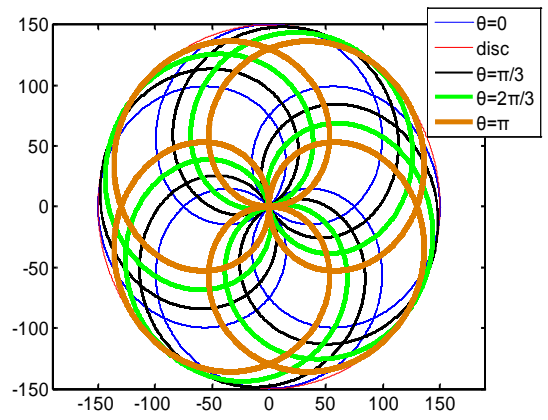

$n=5 / 4$

Fig. 5 Wafer point path on the disc with different circumferential position

The trajectory was investigated in Fig. 5. The initial phase angle $\theta$ of wafer point does not affect the lapping trajectory shape, trajectory area and trajectory intensive degree, only makes the phase of the lapping trajectory change. The disc trajectory phase was affected by the RSR, $n$, and the initial phase angle, $\theta$. For a certain RSR $n$, the lapping trajectory of the wafer point with initial phase angle $\theta$, corresponds to the angle of $n^{*} \theta$ that the trajectory turns to. When the RSR is positive, the direction of trajectory rotation is anticlockwise. Inversely the RSR is negative, the direction of trajectory rotation is clockwise. 


\section{Conclusion}

The research shows the main reason for causing the non-uniformity of disc wear in the lapping process, which is the distribution non-uniformity of wear trajectory on the lapping disc. Furthermore, the distribution uniformity of wear trajectory is influenced by the processing parameters. The results can be summarized as follows.

1) The RSR affected the complexity of the wear trajectory. When the RSR is an integer, the RSR determines the number of petals, and the number of the outermost petal is one. When the RSR is a fraction, the denominator of RSR fraction determines the number of the outermost petals, the numerator of RSR fraction determines the number of petal layers, and the trajectory is denser and more complex, the track overlap degree is lower, the disc trajectory distribution uniformity is better, compared with integral RSR.

2) In different eccentric distance, the lapping trajectory was distributed in the annular region whose width is $2 * r_{A}$. Wafer point radius, $r_{A}$, is larger, the width of lapping path region is larger and the area of lapping path region is larger.

3) The circumferential position affected the distribution location of the wear trajectory. The circumferential position makes the phase of disc wear trajectory change.

4) The disc trajectory phase was affected by the RSR, n, and the initial phase angle, $\theta$. For a certain RSR, the lapping trajectory of the wafer point with initial phase angle $\theta$, corresponds to the angle of $n^{*} \theta$ that the trajectory turns to.

\section{Acknowledgments}

This research was supported by National Natural Science Foundation of China (51305145, 51575197), the Project of Natural Science Foundation of Fujian Province (2016J01235) and Postgraduate Research Innovation Ability Development Plan of Huaqiao University.

\section{References}

[1] Z.W. Zhong, Y.B. Tian, Y. Ang, H. Wu, Optimization of the chemical mechanical polishing process for optical silicon substrates, Int. J. Adv. Manuf. Technol. 60.9-12 (2012) 1197-1206.

[2] B. Cho, H. Kim, R. Manivannan, D. Moon, J. Park, On the mechanism of material removal by fixed abrasive lapping of various glass substrates, WEAR. 302 (2013) 1334-1339.

[3] A.Y. Jiao, H.J. Quan, Z.Z. Li, Y.H. Zou. Study on improving the trajectory to elevate the surface quality of plane magnetic abrasive finishing, Int. J. Adv. Manuf. Technol. 80 (2015) 9-12.

[4] J.X Su, D.M Guo, R.K Kang, Z.J Jin, X.J Li, Y.B Tian. Modeling and Analyzing on Nonuniformity of Material Removal in Chemical Mechanical Polishing of Silicon Wafer, Materials Science Forum. 471-472 (2004) 26-31.

[5] N.Y. Nguyen, Z.W. Zhong, Y.B. Tian. An analytical investigation of pad wear caused by the conditioner in fixed abrasive chemical-mechanical polishing, Int. J. Adv. Manuf. Technol. 77 (2015) 897-905.

[6] J.L. Yuan, W.F. Yao, P. Zhao, B.H. Lyu, Z.X. Chen, M.P. Zhong. Kinematics and trajectory of both-sides cylindrical lapping process in planetary motion type, Int. J. of Machine Tools and Manufacture. 92 (2015) 60-71.

[7] D.W Zhao, T.Q Wang, Y.Y He, X.C Lu. Kinematic Optimization for Chemical Mechanical Polishing Based On Statistical Analysis of Particle Trajectories, IEEE Transactions on Semiconductor Manufacturing. 26.4 (2013) 556-563.

[8] L.Y. Lu, C. F. Fang, J. Y. Shen, J. Lu, X. P Xu. Analysis of path distribution in lapping and polishing with single fixed abrasive, Key Engineering Materials. 589-590 (2013) 475-479. 